\title{
Stability of Runge-Kutta Methods for Neutral Delay Differential Equations
}

\author{
Liping Wen, Xiong Liu, and Yuexin Yu \\ School of Mathematics and Computational Science, Xiangtan University, Xiangtan, Hunan 411105, China \\ Correspondence should be addressed to Liping Wen; lpwen@xtu.edu.cn
}

Received 24 July 2015; Accepted 1 November 2015

Academic Editor: Francisco R. Villatoro

Copyright (C) 2015 Liping Wen et al. This is an open access article distributed under the Creative Commons Attribution License, which permits unrestricted use, distribution, and reproduction in any medium, provided the original work is properly cited.

This paper is concerned with the numerical stability of a class of nonlinear neutral delay differential equations. The numerical stability results are obtained for $(k, l)$-algebraically stable Runge-Kutta methods when they are applied to this type of problem. Numerical examples are given to confirm our theoretical results.

\section{Introduction}

Let $\langle\cdot, \cdot\rangle$ be an inner product on $\mathbb{C}^{N}$ and let $\|\cdot\|$ be the corresponding norm. Consider the initial value problems (IVPs) of nonlinear neutral delay differential equations (NDDEs) as follows (cf. [1]):

$$
\begin{aligned}
y^{\prime}(t) & =f\left(t, y(t), y\left(t-\tau_{1}\right), y^{\prime}\left(t-\tau_{2}\right)\right), \quad t \geq t_{0}, \\
y(t) & =\varphi(t), \quad t_{0}-\tau \leq t \leq t_{0}
\end{aligned}
$$

where $\tau_{1}, \tau_{2}$ are real positive constants, $\tau=\max \left\{\tau_{1}, \tau_{2}\right\}, \varphi$ : $\left[t_{0}-\tau, t_{0}\right] \rightarrow \mathbb{C}^{N}$ is a continuously differentiable function, and $f:\left[t_{0},+\infty\right) \times \mathbb{C}^{N} \times \mathbb{C}^{N} \times \mathbb{C}^{N} \rightarrow \mathbb{C}^{N}$ is a continuous function satisfying

$$
\begin{aligned}
& \operatorname{Re}\left\langle u_{1}-u_{2}, f\left(t, u_{1}, v, w\right)-f\left(t, u_{2}, v, w\right)\right\rangle \\
& \leq \alpha\left\|u_{1}-u_{2}\right\|^{2}, \quad \forall t \geq t_{0}, u_{1}, u_{2}, v, w \in \mathbb{C}^{N}, \\
& \left\|f\left(t, u, v_{1}, w\right)-f\left(t, u, v_{2}, w\right)\right\| \leq \beta_{1}\left\|v_{1}-v_{2}\right\|, \\
& \forall t \geq t_{0}, u, v_{1}, v_{2}, w \in \mathbb{C}^{N}, \\
& \left\|f\left(t, u, v, w_{1}\right)-f\left(t, u, v, w_{2}\right)\right\| \leq \beta_{2}\left\|w_{1}-w_{2}\right\|, \\
& \forall t \geq t_{0}, u, v, w_{1}, w_{2} \in \mathbb{C}^{N},
\end{aligned}
$$

$$
\begin{aligned}
& \left\|F\left(t, u, v_{1}, x_{1}, y, z\right)-F\left(t, u, v_{2}, x_{2}, y, z\right)\right\| \\
& \leq \gamma_{1}\left\|v_{1}-v_{2}\right\|+\gamma_{2}\left\|x_{1}-x_{2}\right\|, \\
& \forall t \geq t_{0}, u, v_{1}, v_{2}, x_{1}, x_{2}, y, z \in \mathbb{C}^{N},
\end{aligned}
$$

where

$$
F(t, u, v, x, y, z):=f\left(t, u, v, f\left(t-\tau_{2}, x, y, z\right)\right)
$$

and $\alpha, \beta_{1}, \beta_{2}, \gamma_{1}$, and $\gamma_{2}$ are real constants and $\beta_{1} \geq 0,0 \leq$ $\beta_{2}<1, \gamma_{1}>0$, and $\gamma_{2}>0$.

The existence and uniqueness of solution to (1) are discussed in $[2,3]$. Throughout this paper we assume that problem (1) has a unique true solution $y(t)$, and we will still use the symbol $\mathbb{D}\left(\alpha, \beta_{1}, \beta_{2}, \gamma_{1}, \gamma_{2}\right)$ presented in literature [1] to denote the problem class consisting of all problem (1) satisfying conditions (2)-(5).

In order to investigate the stability of NDDEs, we have to introduce the perturbed problem of (1), which is defined by the same function $f(t, u, v, w)$, but with another initial condition:

$$
\begin{aligned}
z^{\prime}(t) & =f\left(t, z(t), z\left(t-\tau_{1}\right), z^{\prime}\left(t-\tau_{2}\right)\right), \quad t \geq t_{0}, \\
z(t) & =\psi(t), \quad t_{0}-\tau \leq t \leq t_{0},
\end{aligned}
$$

where $\psi:\left[t_{0}-\tau, t_{0}\right] \rightarrow \mathbb{C}^{N}$ is a continuous and differentiable function. The unique exact solution of problem (7) is denoted by $z(t)$. 
Remark 1. In 2004, Wang and Li [4] considered nonlinear NDDEs of form as

$$
\begin{aligned}
y^{\prime}(t) & =f\left(t, y(t), y(t-\tau), y^{\prime}(t-\tau)\right), \quad t \geq 0, \\
y(t) & =\varphi(t), \quad-\tau \leq t \leq 0,
\end{aligned}
$$

and they obtained a series of stability results of theoretical solution and numerical solution which was given by backward Euler methods. Recently, they further investigated the stability of one-leg methods [5], Runge-Kutta methods [6], and continuous Runge-Kutta-type methods [7] for the solution to problem (8), respectively. More research in this field can be found in literatures [2, 3, 8-15]. The research of numerical methods for more extensive problem class can be seen in [16-19].

The difference between (1) and (8) is that the quantities $\tau_{1}, \tau_{2}$ in (1) can be unequal. However, many real-world phenomena can be described by (1) but not with (8). For the application of this type in the real world, one is referred to [1], because, in these problems, the change of the state variable depends on the state of the past some time and, in addition, a state change of another some time (cf. $[20,21])$. It is easy to see that the existent analytic and numerical stability results for (8) in the abovementioned literatures can not be applied to problem (1).

Remark 2. In 2012, the authors of the present paper considered problem (1), and a sufficient condition for the stability of the problem itself is given [1]. This result is described below.

Theorem 3 (cf. [1]). Assume that (1) belongs to $\mathbb{D}\left(\alpha, \beta_{1}, \beta_{2}\right.$, $\left.\gamma_{1}, \gamma_{2}\right)$ with $\alpha<0$ and

$$
\frac{\gamma_{1}+\gamma_{2}-\beta_{2} \alpha}{-\alpha} \leq 1
$$

Then one has

$$
\|y(t)-z(t)\| \leq \phi_{1}+\frac{\beta_{1} \phi_{1}+\beta_{2} \phi_{2}}{-\alpha} \sum_{i=0}^{\left\lfloor\tau_{2} / \tau_{1}\right\rfloor}\left(1+\frac{\beta_{1}}{-\alpha}\right)^{i},
$$

where

$$
\begin{aligned}
& \phi_{1}=\sup _{t_{0}-\tau \leq s \leq t_{0}}\|\varphi(s)-\psi(s)\|, \\
& \phi_{2}=\sup _{t_{0}-\tau \leq s \leq t_{0}}\left\|\varphi^{\prime}(s)-\psi^{\prime}(s)\right\|,
\end{aligned}
$$

and the notation $\lfloor x\rfloor$ denotes the largest integer smaller than or equal to $x$.

Remark 4. In [1], the numerical stability results are obtained for $A$-stable one-leg methods when they are applied to problem (1). This paper pursues this and further investigates the stability of Runge-Kutta methods for problem (1). It is well known that the Runge-Kutta-type methods are a class of important and common numerical methods for solving differential equations. Therefore, it is important to analyse whether or not Runge-Kutta methods inherit the stability of the underlying problem when they are applied to (1). This also is the motivation of this paper.
The organization of this paper is as follows. In Section 2, the numerical stability results of $(k, l)$-algebraically stable Runge-Kutta methods for (1) are given. Finally, in Section 3, some numerical experiments are given which confirm the theoretical results obtained in this paper.

\section{The Stability of Runge-Kutta Methods}

The adaptation of the $s$ stage Runge-Kutta method

$$
(A, b, c)=\begin{array}{c|cccc}
c_{1} & a_{11} & a_{12} & \cdots & a_{1 s} \\
c_{2} & a_{21} & a_{22} & \cdots & a_{2 s} \\
\vdots & \cdots & \cdots & \cdots & \cdots \\
c_{s} & a_{s 1} & a_{s 2} & \cdots & a_{s s} \\
\hline & b_{1} & b_{2} & \cdots & b_{s}
\end{array}
$$

for ODEs can generally lead to an $s$ stage Runge-Kutta method

$$
\begin{array}{r}
Y_{i}^{(n)}=y_{n}+h \sum_{j=1}^{s} a_{i j} f\left(t_{n}+c_{j} h, Y_{j}^{(n)}, \bar{Y}_{j}^{(n)}, \widetilde{Y}_{j}^{(n)}\right), \\
i=1,2, \ldots, s, \\
y_{n+1}=y_{n}+h \sum_{j=1}^{s} b_{j} f\left(t_{n}+c_{j} h, Y_{j}^{(n)}, \bar{Y}_{j}^{(n)}, \widetilde{Y}_{j}^{(n)}\right), \\
n=0,1, \ldots
\end{array}
$$

for solving problem (1) in NDDEs; here, $h>0$ is the step size and $t_{n}=t_{0}+n h$. The values $y_{n}, Y_{i}^{(n)}, \bar{Y}_{i}^{(n)}$, and $\widetilde{Y}_{i}^{(n)}$ are approximations to $y\left(t_{n}\right), y\left(t_{n}+c_{i} h\right), y\left(t_{n}+c_{i} h-\tau_{1}\right)$, and $y^{\prime}\left(t_{n}+\right.$ $\left.c_{i} h-\tau_{2}\right)$, respectively. $y_{0}=\varphi\left(t_{0}\right)$. The symbols $A$ and $b$ are linear mappings corresponding to the matrices $A=\left[a_{i j}\right] \epsilon$ $R^{s \times s}$ and $b^{T}=\left[b_{1}, b_{2}, \ldots, b_{s}\right] \in R^{s}$, respectively. We always assume that $\sum_{i=1}^{s} b_{i}=1$; each $c_{i}=\sum_{j=1}^{s} a_{i j} \in[0,1]$.

In this paper, we always let $h=\tau_{2} / m_{2}$ for some positive integer $m_{2}$ and $\tau_{1}=\left(m_{1}-\delta\right) h$ with integer $m_{1} \geq 1$ and $\delta \epsilon$ $[0,1)$. Let $Y_{j}^{(i)}=\varphi\left(t_{i}+c_{j} h\right)$ for $i<0,1 \leq j \leq s$. Define (cf. [22])

$$
\bar{Y}_{j}^{(n)}= \begin{cases}\sum_{i=-\mu}^{v} L_{i}(\delta) Y_{j}^{\left(n-m_{1}+i\right)}, & t_{n}+c_{j} h-\tau_{1}>t_{0}, \\ \varphi\left(t_{n}+c_{j} h-\tau_{1}\right), & t_{n}+c_{j} h-\tau_{1} \leq t_{0}, \\ j=1,2, \ldots, s,\end{cases}
$$

where

$$
L_{i}(x)=\prod_{k=-\mu, k \neq i}^{v} \frac{x-k}{i-k}, \quad x \in[0,1)
$$

and we assume integers $\mu, v \geq 0$ and $m_{1} \geq \nu+1$ so as to guarantee that, in the interpolation procedure for $\bar{Y}_{j}^{(n)}$, no unknown values $Y_{j}^{(k)}$ with $k \geq n$ are used. 
The arguments $\widetilde{Y}_{i}^{(n)}$ are given by

$$
\tilde{Y}_{i}^{(n)}=\left\{\begin{array}{ll}
f\left(t_{n}+c_{i} h-\tau_{2}, Y_{i}^{\left(n-m_{2}\right)}, \bar{Y}_{i}^{\left(n-m_{2}\right)}, \tilde{Y}_{i}^{\left(n-m_{2}\right)}\right), & t_{n}+c_{i} h-\tau_{2}>t_{0}, \\
\varphi^{\prime}\left(t_{n}+c_{i} h-\tau_{2}\right), & t_{n}+c_{i} h-\tau_{2} \leq t_{0},
\end{array} \quad i=1,2, \ldots, s .\right.
$$

Similarly, the adaptation of the Runge-Kutta method $(A, b, c)$ with the same interpolation procedures to problem (7) leads to the following process:

$$
\begin{array}{r}
Z_{i}^{(n)}=z_{n}+h \sum_{j=1}^{s} a_{i j} f\left(t_{n}+c_{j} h, Z_{j}^{(n)}, \bar{Z}_{j}^{(n)}, \widetilde{Z}_{j}^{(n)}\right), \\
i=1,2, \ldots, s, \\
z_{n+1}=z_{n}+h \sum_{j=1}^{s} b_{j} f\left(t_{n}+c_{j} h, Z_{j}^{(n)}, \bar{Z}_{j}^{(n)}, \widetilde{Z}_{j}^{(n)}\right), \\
n=0,1, \ldots,
\end{array}
$$

where $z_{0}=\psi\left(t_{0}\right)$.

Definition 5 (cf. [23]). Let $k, l$ be real constants. A method $(A, b, c)$ is said to be $(k, l)$-algebraically stable if there exists a diagonal nonnegative matrix $D=\operatorname{diag}\left(d_{1}, d_{2}, \ldots, d_{s}\right)$ such that $M=\left[M_{i j}\right]$ is nonnegative definite, where

$$
\begin{aligned}
& M \\
& =\left(\begin{array}{cc}
k-1-2 l e^{T} D e & e^{T} D-b^{T}-2 l e^{T} D A \\
D e-b-2 l A^{T} D e & D A+A^{T} D-b b^{T}-2 l A^{T} D A
\end{array}\right) .
\end{aligned}
$$

As an important special case, a $(1,0)$-algebraically stable method is called algebraically stable for short.

In the following, we give the main result of this paper and its proof.

Theorem 6. Assume that method (12) is $(k, l)$-algebraically stable with $0<k \leq 1$ and that problems (1) and (7) belong to $\mathbb{D}\left(\alpha, \beta_{1}, \beta_{2}, \gamma_{1}, \gamma_{2}\right)$ with $\alpha<0, \beta_{1} \leq \gamma_{1}$. Then when

$$
\left(2 \alpha+\frac{\gamma_{1}+L_{0}^{2}(\mu+v+1)^{2} \gamma_{1}+2 \gamma_{2}}{1-\beta_{2}}\right) h \leq 2 l,
$$

method (13) with (14) and (16) is stable; that is,

$$
\begin{gathered}
\left\|y_{n}-z_{n}\right\| \leq C \max \left\{\max _{t_{0}-\tau \leq t \leq t_{0}}\|\varphi(t)-\psi(t)\|,\right. \\
\left.\max _{t_{0}-\tau \leq t \leq t_{0}}\left\|\varphi^{\prime}(t)-\psi^{\prime}(t)\right\|\right\},
\end{gathered}
$$

where the constant $C>0$ depends only on $\alpha, \beta_{1}, \beta_{2}, \gamma_{1}, \gamma_{2}, \tau_{1}$, and $\tau_{2}$ and the sequences $\left\{y_{n}\right\}$ and $\left\{z_{n}\right\}$ are two approximation solutions produced by (13) and (17), respectively.
Proof. For any $n>0$, let $q_{i}^{(n)}=\left\lfloor\left(n+c_{i}\right) / m_{2}\right\rfloor, i=1,2, \ldots, s$; then we have

$$
t_{0}+q_{i}^{(n)} \tau_{2} \leq t_{n}+c_{i} h \leq t_{0}+\left(q_{i}^{(n)}+1\right) \tau_{2} .
$$

Let

$$
\begin{aligned}
w_{n}= & y_{n}-z_{n}, \\
W_{i}^{(n)}= & Y_{i}^{(n)}-Z_{i}^{(n)}, \\
\bar{W}_{i}^{(n)}= & \bar{Y}_{i}^{(n)}-\bar{Z}_{i}^{(n)}, \\
Q_{i}^{(n)}= & f\left(t_{n}+c_{i} h, Y_{i}^{(n)}, \bar{Y}_{i}^{(n)}, \widetilde{Y}_{i}^{(n)}\right) \\
& -f\left(t_{n}+c_{i} h, Z_{i}^{(n)}, \bar{Z}_{i}^{(n)}, \widetilde{Z}_{i}^{(n)}\right) .
\end{aligned}
$$

Then from (13) and (17) we obtain that

$$
\begin{aligned}
& W_{i}^{(n)}=w_{n}+h \sum_{j=1}^{s} a_{i j} Q_{j}^{(n)}, \quad i=1,2, \ldots, s, \\
& w_{n+1}=w_{n}+h \sum_{j=1}^{s} b_{j} Q_{j}^{(n)} .
\end{aligned}
$$

As in [23], we can easily obtain that

$$
\begin{aligned}
& \left\|w_{n+1}\right\|^{2}-k\left\|w_{n}\right\|^{2}-2 \sum_{j=1}^{s} d_{j} \operatorname{Re}\left\langle W_{j}^{(n)}, h Q_{j}^{(n)}-l W_{j}^{(n)}\right\rangle \\
& =-\sum_{i=1}^{s+1} \sum_{j=1}^{s+1} M_{i j}\left\langle u_{i}, u_{j}\right\rangle,
\end{aligned}
$$

where

$$
\begin{aligned}
& u_{1}=w_{n}, \\
& u_{i}=h Q_{i-1}^{(n)}, \quad i=2,3, \ldots, s+1 .
\end{aligned}
$$

Noting that the method is $(k, l)$-algebraically stable; then $(24)$ reads

$$
\begin{aligned}
\left\|w_{n+1}\right\|^{2} \leq & k\left\|w_{n}\right\|^{2} \\
& +2 \sum_{j=1}^{s} d_{j} \operatorname{Re}\left\langle W_{j}^{(n)}, h Q_{j}^{(n)}-l W_{j}^{(n)}\right\rangle .
\end{aligned}
$$


It follows from (2) that

$$
\begin{aligned}
2 \operatorname{Re} & \left\langle W_{i}^{(n)}, h Q_{i}^{(n)}\right\rangle \\
& =2 h \operatorname{Re}\left\langle W_{i}^{(n)}, f\left(t_{n}+c_{i} h, Y_{i}^{(n)}, \bar{Y}_{i}^{(n)}, \widetilde{Y}_{i}^{(n)}\right)\right. \\
& \left.-f\left(t_{n}+c_{i} h, Z_{i}^{(n)}, \bar{Z}_{i}^{(n)}, \widetilde{Z}_{i}^{(n)}\right)\right\rangle \leq 2 h \alpha\left\|W_{i}^{(n)}\right\|^{2} \\
& +2 h\left\|W_{i}^{(n)}\right\| \Gamma_{i}^{(n)}
\end{aligned}
$$

where

$$
\begin{aligned}
\Gamma_{i}^{(n)} & =\| f\left(t_{n}+c_{i} h, Z_{i}^{(n)}, \bar{Y}_{i}^{(n)}, \widetilde{Y}_{i}^{(n)}\right) \\
& -f\left(t_{n}+c_{i} h, Z_{i}^{(n)}, \bar{Z}_{i}^{(n)}, \widetilde{Z}_{i}^{(n)}\right) \| .
\end{aligned}
$$

When $t_{n}+c_{i} h \leq t_{0}+\tau_{2}$, from (3), (4), and (16), we have

$$
\Gamma_{i}^{(n)} \leq \beta_{1}\left\|\bar{W}_{i}^{(n)}\right\|+\beta_{2} \phi_{2} .
$$

Otherwise, when $t_{n}+c_{i} h>t_{0}+\tau_{2}$, from (4), (5), and (16), we have

$$
\begin{aligned}
\Gamma_{i}^{(n)} & =\| f\left(t_{n}+c_{i} h, Z_{i}^{(n)}, \bar{Y}_{i}^{(n)},\right. \\
& \left.f\left(t_{n}+c_{i} h-\tau_{2}, Y_{i}^{\left(n-m_{2}\right)}, \bar{Y}_{i}^{\left(n-m_{2}\right)}, \tilde{Y}_{i}^{\left(n-m_{2}\right)}\right)\right)-f\left(t_{n}\right. \\
& +c_{i} h, Z_{i}^{(n)}, \bar{Z}_{i}^{(n)}, \\
& \left.f\left(t_{n}+c_{i} h-\tau_{2}, Z_{i}^{\left(n-m_{2}\right)}, \bar{Y}_{i}^{\left(n-m_{2}\right)}, \widetilde{Y}_{i}^{\left(n-m_{2}\right)}\right)\right)+f\left(t_{n}\right. \\
& +c_{i} h, Z_{i}^{(n)}, \bar{Z}_{i}^{(n)}, \\
& \left.f\left(t_{n}+c_{i} h-\tau_{2}, Z_{i}^{\left(n-m_{2}\right)}, \bar{Y}_{i}^{\left(n-m_{2}\right)}, \widetilde{Y}_{i}^{\left(n-m_{2}\right)}\right)\right)-f\left(t_{n}\right. \\
& +c_{i} h, Z_{i}^{(n)}, \bar{Z}_{i}^{(n)}, \\
& \left.f\left(t_{n}+c_{i} h-\tau_{2}, Z_{i}^{\left(n-m_{2}\right)}, \bar{Z}_{i}^{\left(n-m_{2}\right)}, \widetilde{Z}_{i}^{\left(n-m_{2}\right)}\right)\right) \| \\
& \leq \gamma_{1}\left\|\bar{W}_{i}^{(n)}\right\|+\gamma_{2}\left\|W_{i}^{\left(n-m_{2}\right)}\right\|+\beta_{2} \Gamma_{i}^{\left(n-m_{2}\right)} .
\end{aligned}
$$

From this and by induction we have

$$
\begin{aligned}
\Gamma_{i}^{(n)} \leq & \gamma_{1} \sum_{j=1}^{q_{i}^{(n)}} \beta_{2}^{j-1}\left\|\bar{W}_{i}^{\left(n-(j-1) m_{2}\right)}\right\|+\gamma_{2} \sum_{j=1}^{q_{i}^{(n)}} \beta_{2}^{j-1}\left\|W_{i}^{\left(n-j m_{2}\right)}\right\| \\
& +\beta_{2}^{q_{i}^{(n)}} \Gamma_{i}^{\left(n-q_{i}^{(n)} m_{2}\right)} \\
\leq & \gamma_{1} \sum_{j=1}^{q_{i}^{(n)}+1} \beta_{2}^{j-1}\left\|\bar{W}_{i}^{\left(n-(j-1) m_{2}\right)}\right\| \\
& +\gamma_{2} \sum_{j=1}^{q_{i}^{(n)}} \beta_{2}^{j-1}\left\|W_{i}^{\left(n-j m_{2}\right)}\right\|+\beta_{2}^{q_{i}^{(n)}} \beta_{2} \phi_{2},
\end{aligned}
$$

where we have used (29) and $\beta_{1} \leq \gamma_{1}$. Apparently, inequality (29) can be integrated to (31) by $q_{i}^{(n)}=0$. Note that, here and later, when $q<p$, the value of $\operatorname{sum} \sum_{p}^{q}=0$.

Inequality (27) together with (31) yields

$$
\begin{aligned}
2 \operatorname{Re}\left\langle W_{i}^{(n)}, h Q_{i}^{(n)}\right\rangle \leq & h\left(2 \alpha+\frac{\gamma_{1}+\gamma_{2}}{1-\beta_{2}}\right)\left\|W_{i}^{(n)}\right\|^{2} \\
& +h \gamma_{1} \sum_{j=1}^{q_{i}^{(n)}+1} \beta_{2}^{j-1}\left\|\bar{W}_{i}^{\left(n-(j-1) m_{2}\right)}\right\|^{2} \\
& +h \gamma_{2} \sum_{j=1}^{q_{i}^{(n)}} \beta_{2}^{j-1}\left\|W_{i}^{\left(n-j m_{2}\right)}\right\|^{2} \\
& +h \frac{\beta_{2}^{q_{i}^{(n)}} \beta_{2}^{2}}{\gamma_{2}} \phi_{2}^{2} .
\end{aligned}
$$

Denote

$$
\mu_{1}=h\left(2 \alpha+\frac{\gamma_{1}+\gamma_{2}}{1-\beta_{2}}\right)-2 l .
$$

Then, from (26) and noting that $0<k \leq 1$, we can easily obtain

$$
\begin{aligned}
& \left\|w_{n+1}\right\|^{2} \leq k\left\|w_{n}\right\|^{2}+\mu_{1} \sum_{i=1}^{s} d_{i}\left\|W_{i}^{(n)}\right\|^{2} \\
& +h \sum_{i=1}^{s} d_{i}\left(\gamma_{1} \sum_{j=1}^{q_{i}^{(n)}+1} \beta_{2}^{j-1}\left\|\bar{W}_{i}^{\left(n-(j-1) m_{2}\right)}\right\|^{2}\right. \\
& \left.+\gamma_{2} \sum_{j=1}^{q_{i}^{(n)}} \beta_{2}^{j-1}\left\|W_{i}^{\left(n-j m_{2}\right)}\right\|^{2}+\frac{\beta_{2}^{q_{i}^{(n)}} \beta_{2}^{2}}{\gamma_{2}} \phi_{2}^{2}\right) \leq\left\|w_{0}\right\|^{2} \\
& +\mu_{1} \sum_{j=0}^{n} \sum_{i=1}^{s} d_{i}\left\|W_{i}^{(j)}\right\|^{2} \\
& +h \gamma_{1} \sum_{\mu=0}^{n} \sum_{i=1}^{s} d_{i} \sum_{j=1}^{(\mu)}+1 \beta_{2}^{j-1}\left\|\bar{W}_{i}^{\left(\mu-(j-1) m_{2}\right)}\right\|^{2} \\
& +h \gamma_{2} \sum_{\mu=0}^{n} \sum_{i=1}^{s} d_{i} \sum_{j=1}^{q_{i}^{(\mu)}} \beta_{2}^{j-1}\left\|W_{i}^{\left(\mu-j m_{2}\right)}\right\|^{2} \\
& +h \sum_{\mu=0}^{n} \sum_{i=1}^{s} d_{i} \beta_{2}^{q_{i}^{(\mu)}} \frac{\beta_{2}^{2}}{\gamma_{2}} \phi_{2}^{2} \leq\left\|w_{0}\right\|^{2} \\
& +\mu_{1} \sum_{i=1}^{s} d_{i} \sum_{j=0}^{n}\left\|W_{i}^{(j)}\right\|^{2}+\frac{h \gamma_{2}}{1-\beta_{2}} \sum_{i=1}^{s} d_{i} \sum_{j=0}^{n}\left\|W_{i}^{(j)}\right\|^{2} \\
& +\frac{h \gamma_{1}}{1-\beta_{2}} \sum_{i=1}^{s} d_{i} \sum_{j=0}^{n}\left\|\bar{W}_{i}^{(j)}\right\|^{2}+\frac{h d m_{2}}{1-\beta_{2}} \frac{\beta_{2}^{2}}{\gamma_{2}} \phi_{2}^{2} \leq\left\|w_{0}\right\|^{2}
\end{aligned}
$$


Discrete Dynamics in Nature and Society

5

$$
\begin{aligned}
& +\left(\mu_{1}+\frac{h \gamma_{2}}{1-\beta_{2}}\right) \sum_{i=1}^{s} d_{i} \sum_{j=0}^{n}\left\|W_{i}^{(j)}\right\|^{2}+\frac{h \gamma_{1}}{1-\beta_{2}} \\
& \cdot \sum_{i=1}^{s} d_{i} \sum_{j=0}^{n}\left\|\bar{W}_{i}^{(j)}\right\|^{2}+\frac{d \tau_{2}}{1-\beta_{2}} \frac{\beta_{2}^{2}}{\gamma_{2}} \phi_{2}^{2},
\end{aligned}
$$

where $d=\sum_{i=1}^{s} d_{i}$.

In order to estimate the term $\left\|\bar{W}_{i}^{(j)}\right\|$ in (34), we denote $J_{0}=\{1,2, \ldots, s\}, J_{1}=\left\{j \in J_{0} \mid c_{j}+\delta \leq 1\right\}$. Then when $n \geq m_{1}$ or $n=m_{1}-1$ but $j \in J_{0}-J_{1}$, from (14), we have

$$
\begin{aligned}
\left\|\bar{W}_{j}^{(n)}\right\| & =\left\|\bar{Y}_{j}^{(n)}-\bar{Z}_{j}^{(n)}\right\| \\
& =\left\|\sum_{i=-\mu}^{v} L_{i}(\delta)\left(Y_{j}^{\left(n-m_{1}+i\right)}-Z_{j}^{\left(n-m_{1}+i\right)}\right)\right\| \\
& \leq L_{0} \sum_{i=-\mu}^{v}\left\|W_{j}^{\left(n-m_{1}+i\right)}\right\|
\end{aligned}
$$

where

$$
L_{0}=\max _{-\mu \leq i \leq v} \sup _{x \in[0,1)}\left|L_{i}(x)\right|
$$

Therefore, we obtain that

$$
\left\|\bar{W}_{j}^{(n)}\right\|^{2} \leq L_{0}^{2}(\mu+\nu+1) \sum_{i=-\mu}^{\nu}\left\|W_{j}^{\left(n-m_{1}+i\right)}\right\|^{2} .
$$

Otherwise, when $n \leq m_{1}-2$ or $n=m_{1}-1$ but $j \in J_{1}$, we have

$$
\left\|\bar{W}_{j}^{(n)}\right\| \leq \phi_{1}
$$

Therefore, when $n \leq m_{1}-2$, (34) with (38) gives

$$
\begin{gathered}
\left\|w_{n+1}\right\|^{2} \leq\left\|w_{0}\right\|^{2}+\left(\mu_{1}+\frac{h \gamma_{2}}{1-\beta_{2}}\right) \sum_{i=1}^{s} d_{i} \sum_{j=0}^{n}\left\|W_{i}^{(j)}\right\|^{2} \\
+\frac{h \gamma_{1}}{1-\beta_{2}} \sum_{i=1}^{s} d_{i} \sum_{j=0}^{n} \phi_{1}^{2}+\frac{d \tau_{2}}{1-\beta_{2}} \frac{\beta_{2}^{2}}{\gamma_{2}} \phi_{2}^{2} \\
\leq\left\|w_{0}\right\|^{2}+\left(\mu_{1}+\frac{h \gamma_{2}}{1-\beta_{2}}\right) \sum_{i=1}^{s} d_{i} \sum_{j=0}^{n}\left\|W_{i}^{(j)}\right\|^{2} \\
+\frac{d \tau_{1} \gamma_{1}}{1-\beta_{2}} \phi_{1}^{2}+\frac{d \tau_{2}}{1-\beta_{2}} \frac{\beta_{2}^{2}}{\gamma_{2}} \phi_{2}^{2} .
\end{gathered}
$$

When $n=m_{1}-1$, (34) gives

$$
\begin{aligned}
\left\|w_{m_{1}}\right\|^{2} & \left\|w_{0}\right\|^{2}+\left(\mu_{1}+\frac{h \gamma_{2}}{1-\beta_{2}}\right) \sum_{i=1}^{s} d_{i} \sum_{j=0}^{m_{1}-1}\left\|W_{i}^{(j)}\right\|^{2} \\
& +\frac{h \gamma_{1}}{1-\beta_{2}} \sum_{i=1}^{s} d_{i} \sum_{j=0}^{m_{1}-1}\left\|\bar{W}_{i}^{(j)}\right\|^{2}+\frac{d \tau_{2}}{1-\beta_{2}} \frac{\beta_{2}^{2}}{\gamma_{2}} \phi_{2}^{2} \\
\leq & \left\|w_{0}\right\|^{2}+\left(\mu_{1}+\frac{h \gamma_{2}}{1-\beta_{2}}\right) \sum_{i=1}^{s} d_{i} \sum_{j=0}^{m_{1}-1}\left\|W_{i}^{(j)}\right\|^{2} \\
& +\frac{2 d \tau_{1} \gamma_{1}}{1-\beta_{2}} \phi_{1}^{2}+\frac{d \tau_{2}}{1-\beta_{2}} \frac{\beta_{2}^{2}}{\gamma_{2}} \phi_{2}^{2} \\
& +\frac{h \gamma_{1}}{1-\beta_{2}} \sum_{i \in J_{0}-J_{1}} d_{i}\left\|\bar{W}_{i}^{\left(m_{1}-1\right)}\right\|^{2} \\
& +\frac{2 d \tau_{1} \gamma_{1}}{1-\beta_{2}} \phi_{1}^{2}+\frac{d \tau_{2}}{1-\beta_{2}} \frac{\beta_{2}^{2}}{\gamma_{2}} \phi_{2}^{2}\left\|^{2}+\left(\mu_{1}+\frac{h \gamma_{2}}{1-\beta_{2}}\right) \sum_{i=1}^{s} d_{i} \sum_{j=0}^{m_{1}-1}\right\| W_{i}^{(j)} \|^{2} \\
\leq & \left\|w_{0}\right\|^{2}+\left(\mu_{1}+\frac{h \gamma_{2}}{1-\beta_{2}}\right) \sum_{i=1}^{s} d_{i} d_{j=0}^{m_{1}-1}\left\|W_{i}^{(j)}\right\|^{2}\left\|W_{i}^{(-1+j)}\right\|^{2} \\
+ & \frac{2 d \tau_{1} \gamma_{1}}{1-\beta_{2}} \phi_{1}^{2}+\frac{d \tau_{2}}{1-\beta_{2}} \frac{\beta_{2}^{2}}{\gamma_{2}} \phi_{2}^{2} \\
&
\end{aligned}
$$

When $n \geq m_{1}$, from (34) and (37) we have

$$
\begin{aligned}
& \left\|w_{n+1}\right\|^{2} \leq\left\|w_{0}\right\|^{2}+\left(\mu_{1}+\frac{h \gamma_{2}}{1-\beta_{2}}\right) \sum_{i=1}^{s} d_{i} \sum_{j=0}^{n}\left\|W_{i}^{(j)}\right\|^{2} \\
& +\frac{2 d \tau_{1} \gamma_{1}}{1-\beta_{2}} \phi_{1}^{2}+\frac{d \tau_{2}}{1-\beta_{2}} \frac{\beta_{2}^{2}}{\gamma_{2}} \phi_{2}^{2}+L_{0}^{2}(\mu+\nu+1) \frac{h \gamma_{1}}{1-\beta_{2}} \\
& +\sum_{k=m_{1}-1}^{n} \sum_{i=1}^{s} d_{i} \sum_{j=-\mu}^{v}\left\|W_{i}^{\left(k-m_{1}+j\right)}\right\|^{2} \leq\left\|w_{0}\right\|^{2} \\
& +\left(h\left(2 \alpha+\frac{\gamma_{1}+L_{0}^{2}(\mu+\nu+1)^{2} \gamma_{1}+2 \gamma_{2}}{1-\beta_{2}}\right)-2 l\right) \\
& \cdot \sum_{i=1}^{s} d_{i} \sum_{j=0}^{n}\left\|W_{i}^{(j)}\right\|^{2}
\end{aligned}
$$




$$
\begin{aligned}
& +\left(2+L_{0}^{2}(\mu+\nu+1) \frac{(\mu+1)(\mu+2)}{2}\right) \frac{d \tau_{1} \gamma_{1}}{1-\beta_{2}} \phi_{1}^{2} \\
& +\frac{d \tau_{2}}{1-\beta_{2}} \frac{\beta_{2}^{2}}{\gamma_{2}} \phi_{2}^{2} .
\end{aligned}
$$

By condition (19), a combination of (39), (40), and (41) shows the method is stable, which completes the proof of Theorem 6.

For the case where the Runge-Kutta method is algebraically stable, from Theorem 6 we can obtain following result.

Corollary 7. Assume that method (12) is algebraically stable and that problems (1) and (7) belong to $\mathbb{D}\left(\alpha, \beta_{1}, \beta_{2}, \gamma_{1}, \gamma_{2}\right)$ with $\alpha<0, \beta_{1} \leq \gamma_{1}$. Then when

$$
2 \alpha+\frac{\gamma_{1}\left(1+L_{0}^{2}(\mu+\nu+1)^{2}\right)+2 \gamma_{2}}{1-\beta_{2}} \leq 0,
$$

method (13) with (14) and (16) is unconditionally stable for step size $h$.

Remark 8. It is well known that the Gauss, Radau IA, Radau IIA, and Lobatto IIIC Runge-Kutta methods are all algebraically stable; therefore, from Corollary 7, these methods with (14) and (16) are stable when applied to (1) $\epsilon$ $\mathbb{D}\left(\alpha, \beta_{1}, \beta_{2}, \gamma_{1}, \gamma_{2}\right)$ with $\alpha<0, \beta_{1} \leq \gamma_{1}$ provided (42) satisfied. Paper [24] also shows that the Gauss and Radau methods have some good stability properties for delay equations. This is in harmony with the results in this paper.

Remark 9. When $\tau_{1}=\tau_{2}$ in right-hand side function of problem (1), from Theorem 6 we can obtain the corresponding stability result which is similar to that obtained by Wang in [6], but the result of Theorem 6 is more extensive than that in [6], because, in [6], for processing the delay items, only piecewise linear interpolation is considered, which will lead to higher order method appear order reduction phenomenon for the problem which possesses sufficiently smooth solution.

Remark 10. For the method which has order at most 2, we can choose $v=1, \mu=0$ in (15) to process $\bar{Y}_{j}^{(n)}$. Here (14) can be reduced to

$$
\begin{array}{r}
\bar{Y}_{j}^{(n)} \\
=\left\{\begin{array}{lr}
(1-\delta) Y_{j}^{\left(n-m_{1}\right)}+\delta Y_{j}^{\left(n-m_{1}+1\right)}, & t_{n}+c_{j} h-\tau_{1}>t_{0}, \\
\varphi\left(t_{n}+c_{j} h-\tau_{1}\right), & t_{n}+c_{j} h-\tau_{1} \leq t_{0}, \\
& j=1,2, \ldots, s,
\end{array}\right.
\end{array}
$$

which is widely used by many authors for numerically solving differential equations with delay term, such as $[6,8,25]$. Theorem 6 and Corollary 7 are suitable for this situation certainly. Notable is, in this case, the conclusion of the theorem can be more perfect, if we deduce it directly based on (43).

\section{Numerical Experiment}

We consider the following nonlinear NDDE:

$$
\begin{aligned}
y_{1}^{\prime}(t)= & -6 y_{1}(t)+0.1 \sin y_{2}(t-1) \sin y_{1}^{\prime}(t-0.33) \\
& +\phi_{1}(t), \quad t \geq 0 \\
y_{2}^{\prime}(t)= & -4 y_{2}(t)-0.2 \cos y_{1}(t-1) \cos y_{2}^{\prime}(t-0.33) \\
& +\phi_{2}(t), \quad t \geq 0 \\
y_{1}(t)= & \sin 5 t \\
y_{2}(t)= & \cos 5 t
\end{aligned}
$$

$$
-1 \leq t \leq 0
$$

where

$$
\begin{aligned}
& \phi_{1}(t) \\
& =5 \cos 5 t+6 \sin 5 t \\
& -0.1 \sin (\cos 5(t-1)) \sin (5 \cos 5(t-0.33)), \\
& \phi_{2}(t) \\
& =-5 \sin 5 t+4 \cos 5 t \\
& +0.2 \cos (\sin 5(t-1)) \cos (5 \sin 5(t-0.33)) \text {. }
\end{aligned}
$$

Let $\alpha=-4, \beta_{1}=0.2, \beta_{2}=0.2, \gamma_{1}=0.2$, and $\gamma_{2}=1.2$; then problem (44) belongs to $\mathbb{D}\left(\alpha, \beta_{1}, \beta_{2}, \gamma_{1}, \gamma_{2}\right)$ and condition (9) is satisfied, where the inner product is standard inner product. Theorem 3 implies that system (44) is stable.

In order to solve problem (44), we consider the 2-stage Radau IIA Runge-Kutta method:

$$
\begin{aligned}
Y_{1}^{(n)}= & y_{n}+\frac{5}{12} h f\left(t_{n}+\frac{1}{3} h, Y_{1}^{(n)}\right) \\
& -\frac{1}{12} h f\left(t_{n}+h, Y_{2}^{(n)}\right), \\
Y_{2}^{(n)}= & y_{n}+\frac{3}{4} h f\left(t_{n}+\frac{1}{3} h, Y_{1}^{(n)}\right) \\
& +\frac{1}{4} h f\left(t_{n}+h, Y_{2}^{(n)}\right), \\
y_{n+1}= & Y_{2}^{(n)} .
\end{aligned}
$$

Because method (46) is $A$-stable, then from Theorem 6 the numerical solutions will preserve the stability of problem (44) itself. Let $h=0.033$ and $h=0.0033$; the numerical solutions $y_{1 n}, y_{2 n}$ and $z_{1 n}, z_{2 n}$ are computed, and the errors $\left\|y_{n}-z_{n}\right\|$ are shown in Figures 1 and 2, respectively, where $y_{n}=\left[y_{1 n}, y_{2 n}\right]^{T}$ and $z_{n}=\left[z_{1 n}, z_{2 n}\right]^{T}$ are given by applying (46) to (44) and its perturbed problem, which takes initial functions

$$
\begin{aligned}
& y_{1}(t)=\sin (6 t)+0.1, \\
& y_{2}(t)=\cos (4 t)-0.2, \\
& \quad-1 \leq t \leq 0 .
\end{aligned}
$$

These results further confirm our conclusions. 


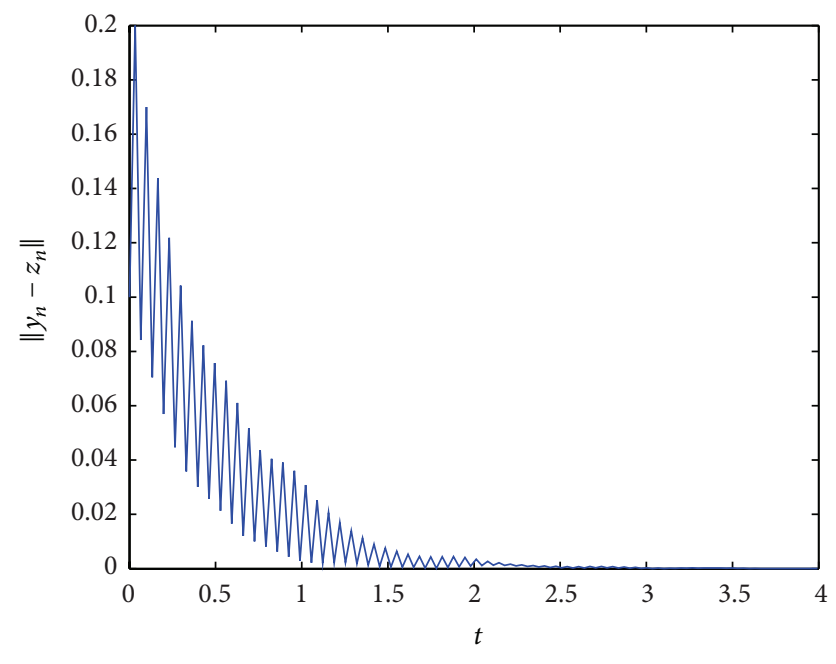

FIgURE 1: The norm of difference $\left\|y_{n}-z_{n}\right\|$ with step size $h=0.033$.

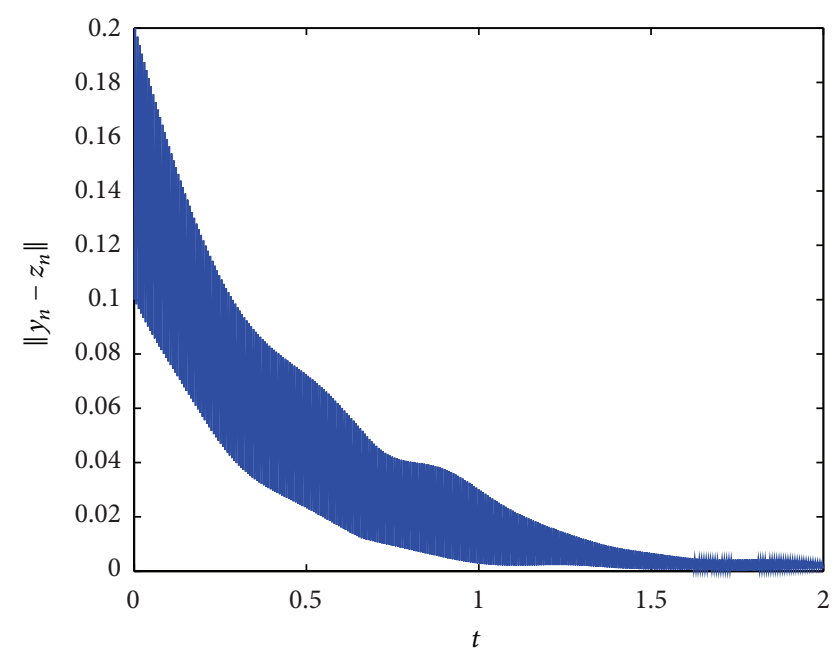

FIGURE 2: The norm of difference $\left\|y_{n}-z_{n}\right\|$ with step size $h=0.0033$.

\section{Conflict of Interests}

The authors declare that there is no conflict of interests regarding the publication of this paper.

\section{Acknowledgments}

This work was supported by the NSF of China (11371302) and Scientific Research Fund of Hunan Provincial Education Department (15A184).

\section{References}

[1] L. Wen and X. Liu, "Numerical stability of one-leg methods for neutral delay differential equations," BIT Numerical Mathematics, vol. 52, no. 1, pp. 251-269, 2012.

[2] A. Bellen, S. Maset, M. Zennaro, and N. Guglielmi, "Recent trends in the numerical solution of retarded functional differential equations," Acta Numerica, vol. 18, pp. 1-110, 2009.
[3] A. Bellen and M. Zennaro, Numerical Methods for Delay Differential Equations, Oxford University Press, Oxford, UK, 2003.

[4] W. S. Wang and S. F. Li, "Stability analysis of nonlinear delay differential equations of neutral type," Mathematica Numerica Sinica, vol. 26, no. 3, pp. 303-314, 2004.

[5] W.-S. Wang, Y. Zhang, and S.-F. Li, "Nonlinear stability of oneleg methods for delay differential equations of neutral type," Applied Numerical Mathematics, vol. 58, no. 2, pp. 122-130, 2008.

[6] W.-S. Wang, S.-F. Li, and K. Su, "Nonlinear stability of RungeKutta methods for neutral delay differential equations," Journal of Computational and Applied Mathematics, vol. 214, no. 1, pp. 175-185, 2008.

[7] W. S. Wang, Y. Zhang, and S. F. Li, "Stability of continuous Runge-Kutta-type methods for nonlinear neutral delaydifferential equations," Applied Mathematical Modelling, vol. 33, no. 8, pp. 3319-3329, 2009.

[8] C.-J. Zhang, "Nonlinear stability of natural Runge-Kutta methods for neutral delay differential equtions," Journal of Computational Mathematics, vol. 20, no. 6, pp. 583-590, 2002.

[9] A. Bellen, N. Guglielmi, and M. Zennaro, "On the contractivity and asymptotic stability of systems of delay differential equations of neutral type," BIT Numerical Mathematics, vol. 39, no. 1, pp. 1-24, 1999.

[10] G.-D. Hu and T. Mitsui, "Stability analysis of numerical methods for systems of neutral delay-differential equations," BIT Numerical Mathematics, vol. 35, no. 4, pp. 504-515, 1995.

[11] J. X. Kuang, J. X. Xiang, and H. J. Tian, "The asymptotic stability of one-parameter methods for neutral differential equations," BIT Numerical Mathematics, vol. 34, no. 3, pp. 400-408, 1994.

[12] L. Qiu, B. Yang, and J. X. Kuang, "The NGP-stability of Runge-Kutta methods for systems of neutral delay differential equations," Numerische Mathematik, vol. 81, no. 3, pp. 451-459, 1999.

[13] T. Koto, "A stability property of A-stable collocation-based Runge-Kutta methods for neutral delay differential equations," BIT Numerical Mathematics, vol. 36, no. 4, pp. 855-859, 1996.

[14] R. Vermiglio and L. Torelli, "A stable numerical approach for implicit non-linear neutral delay differential equations," BIT Numerical Mathematics, vol. 43, no. 1, pp. 195-215, 2003.

[15] C. M. Huang and Q. S. Chang, "Linear stability of general linear methods for systems of neutral delay differential equations," Applied Mathematics Letters, vol. 14, no. 8, pp. 1017-1021, 2001.

[16] L. P. Wen, S. X. Wang, and Y. X. Yu, "Dissipativity of RungeKutta methods for neutral delay integro-differential equations," Applied Mathematics and Computation, vol. 215, no. 2, pp. 583590, 2009.

[17] W. S. Wang, "Nonlinear stability of one-leg methods for neutral Volterra delay-integro-differential equations," Mathematics and Computers in Simulation, vol. 97, pp. 147-161, 2014.

[18] T. T. Qin and C. J. Zhang, "Stable solutions of one-leg methods for a class of nonlinear functional-integro-differential equations," Applied Mathematics and Computation, vol. 250, pp. 4757, 2015.

[19] Y. Yu and L. Wen, "Stability analysis of one-leg methods for nonlinear neutral delay integrodifferential equations," Discrete Dynamics in Nature and Society, vol. 2015, Article ID 325364, 9 pages, 2015.

[20] R. P. Agarwal and S. R. Grace, "Asymptotic stability of certain neutral differential equations," Mathematical and Computer Modelling, vol. 31, no. 8-9, pp. 9-15, 2000. 
[21] J. H. Park, O. M. Kwon, and S. M. Lee, "State estimation for neural networks of neutral-type with interval time-varying delays," Applied Mathematics and Computation, vol. 203, no. 1, pp. 217-223, 2008.

[22] K. J. in't Hout, "A new interpolation procedure for adapting Runge-Kutta methods to delay differential equations," BIT Numerical Mathematics, vol. 32, no. 4, pp. 634-649, 1992.

[23] K. Burrage and J. C. Butcher, "Nonlinear stability of a general class of differential equation methods," BIT Numerical Mathematics, vol. 20, no. 2, pp. 185-203, 1980.

[24] C. Huang, "Delay-dependent stability of high order RungeKutta methods," Numerische Mathematik, vol. 111, no. 3, pp. 377$387,2009$.

[25] C. M. Huang, H. Y. Fu, S. F. Li, and G. N. Chen, "Stability analysis of Runge-Kutta methods for non-linear delay differential equations," BIT. Numerical Mathematics, vol. 39, no. 2, pp. 270280,1999 . 


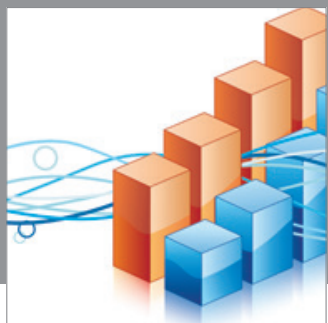

Advances in

Operations Research

mansans

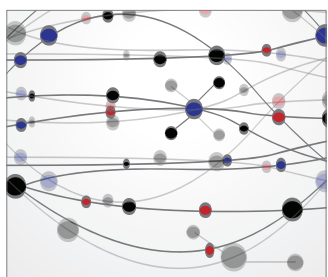

The Scientific World Journal
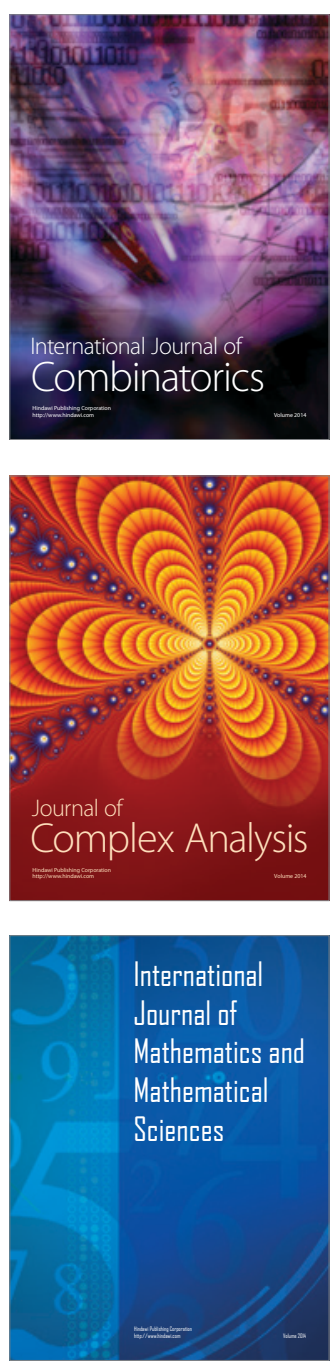
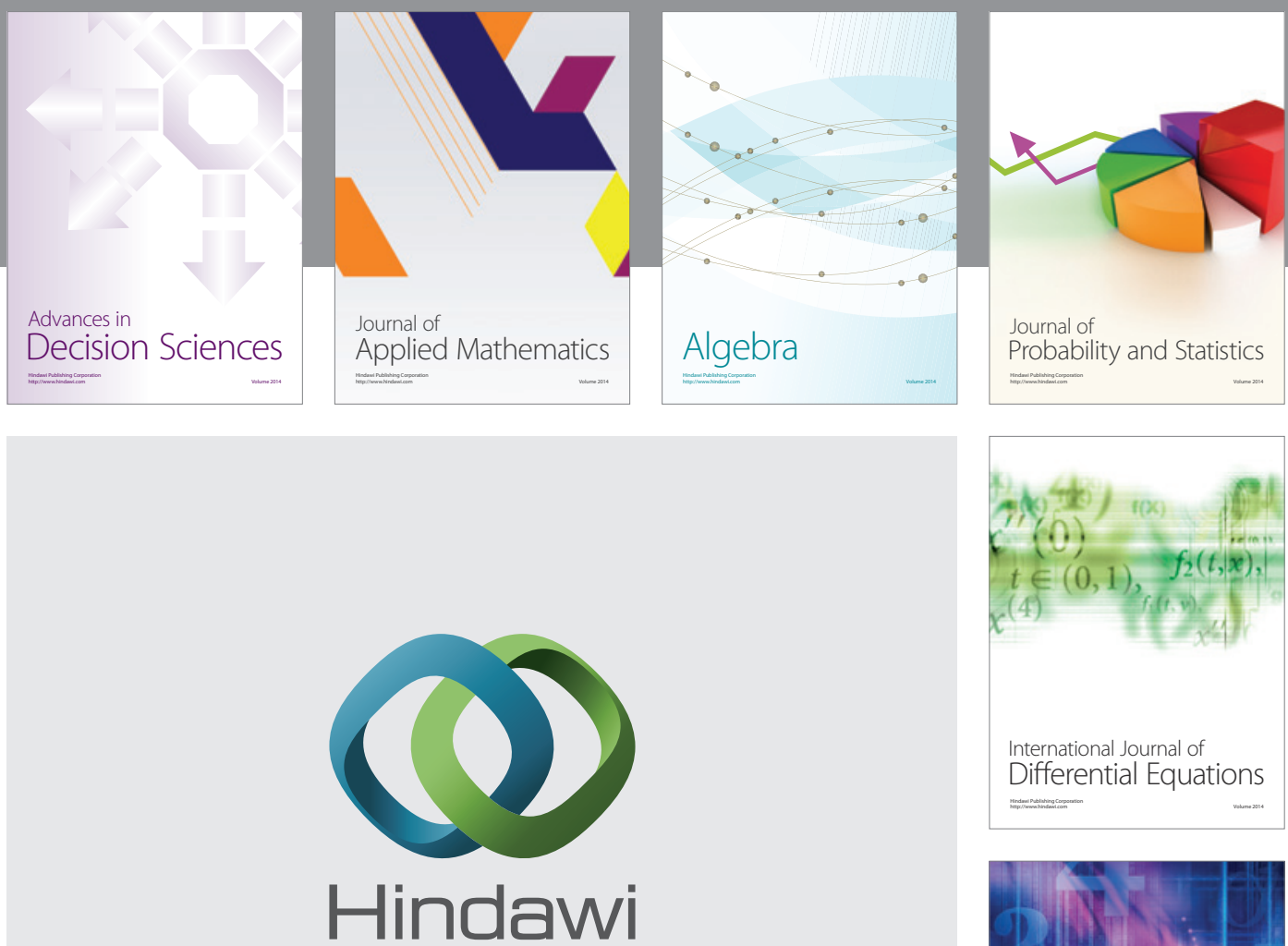

Submit your manuscripts at http://www.hindawi.com
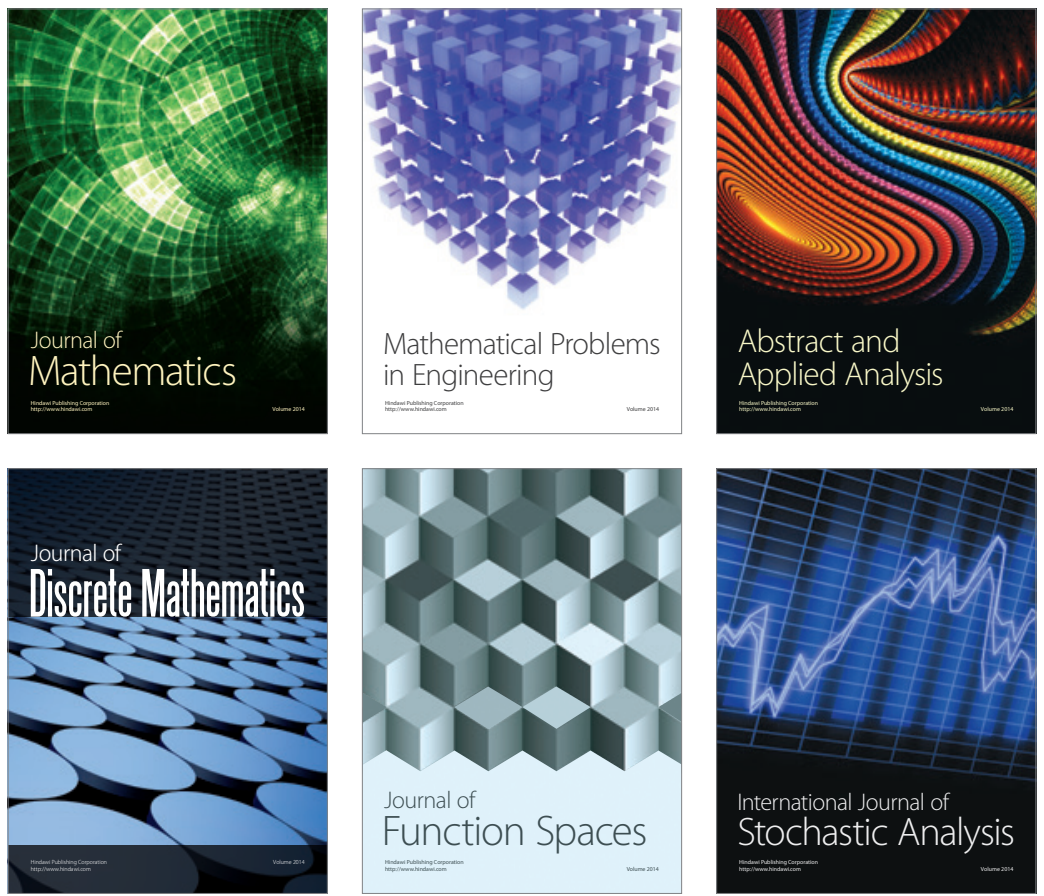

Journal of

Function Spaces

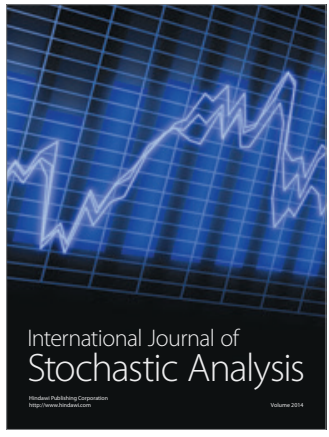

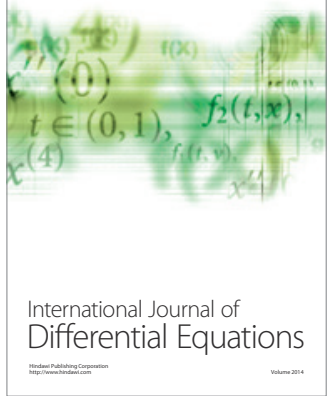
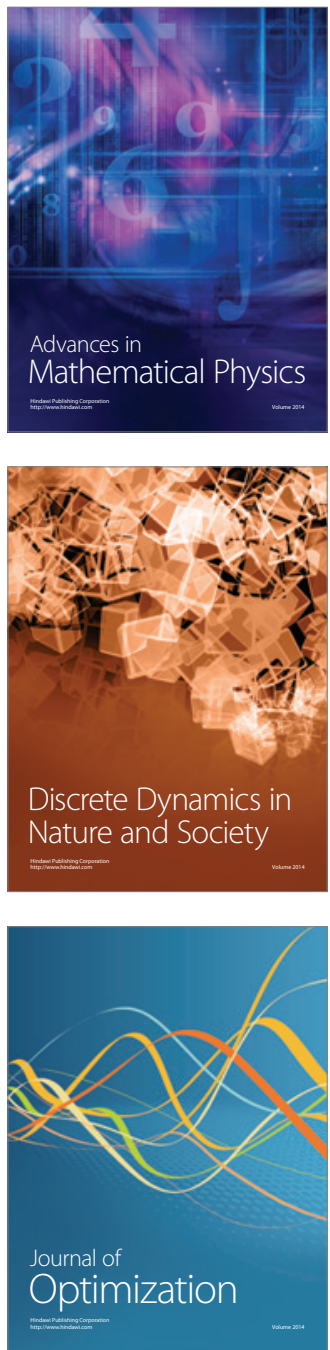